\title{
A Note on Entanglement of Formation and Generalized Concurrence ${ }^{1}$
}

\author{
Shao-Ming Fei ${ }^{\dagger \dagger}$, Zhi-Xi Wang ${ }^{\dagger}$, Hui Zhao ${ }^{\dagger}$ \\ ${ }^{\dagger}$ Department of Mathematics, Capital Normal University, Beijing 100037, P.R. China \\ ${ }^{\ddagger}$ Institut für Angewandte Mathematik, Universität Bonn, 53115 Bonn, Germany
}

\begin{abstract}
We discuss a kind of generalized concurrence for a class of high dimensional quantum pure states such that the entanglement of formation is a monotonically increasing convex function of the generalized concurrence. An analytical expression of the entanglement of formation for a class of high dimensional quantum mixed states is obtained.
\end{abstract}

PACS numbers: 03.65.Bz, 89.70.+c

Key words: Entanglement of formation, Generalized concurrence

Characterized by the simultaneous occurrence of superposition and correlation in composite systems, quantum entanglement has in recent years emerged as the key resource in quantum information processing [1] and resulted in the explosion of interest in quantum computing and communication [2]. To quantify entanglement, a number of entanglement measures has been proposed for bipartite systems. Among them, the pioneering contributions of Bennett et al. [3, 4] defined entanglements of formation and distillation on considerations of convertibility vis-à-vis maximally entangled pairs. The relative entropy of entanglement [5] is based on distinguishability from the set of separable states. Recently there are also discussions of entanglement measure on multipartite case [6].

The entanglement of formation is defined for arbitrary dimensional bipartite systems. Due to the extremizations involved in the calculation, so far no explicit analytic formulae

\footnotetext{
${ }^{1}$ Supported by the NSF of China, Grant No. 10271081.
} 
for entanglement of formation have been found for systems larger than a pair of qubits, except for some special symmetric states [7].

Let $\mathcal{H}$ be an $N$-dimensional complex Hilbert space with orthonormal basis $e_{i}, i=1, \ldots, N$. A pure state on $\mathcal{H} \otimes \mathcal{H}$ is generally of the form,

$$
|\psi\rangle=\sum_{i, j=1}^{N} a_{i j} e_{i} \otimes e_{j}, \quad a_{i j} \in \mathbb{C}
$$

with normalization $\sum_{i, j=1}^{N} a_{i j} a_{i j}^{*}=1$. The entanglement of formation $E$ is defined to be the entropy of either of the two sub-Hilbert space $\mathcal{H} \otimes \mathcal{H}[4]$,

$$
E(|\psi\rangle)=-\operatorname{Tr}\left(\rho_{1} \log _{2} \rho_{1}\right)=-\operatorname{Tr}\left(\rho_{2} \log _{2} \rho_{2}\right)
$$

where $\rho_{1}$ (resp. $\rho_{2}$ ) is the partial trace of $|\psi\rangle\langle\psi|$ over the second (resp. first) Hilbert space of $\mathcal{H} \otimes \mathcal{H}$. Let $A$ denote the matrix with entries given by $a_{i j}$ in (1). $\rho_{1}$ can be expressed as $\rho_{1}=A A^{\dagger}$. A general density matrix $\rho$ on $\mathcal{H} \otimes \mathcal{H}$ has pure-state decompositions of $\left|\psi_{a}\right\rangle$ of the form (1) with probabilities $p_{a}$,

$$
\rho=\sum_{a=1}^{M} p_{a}\left|\psi_{a}\right\rangle\left\langle\psi_{a}\right|, \quad \sum_{a=1}^{M} p_{a}=1
$$

for some $M \in \mathbb{N}$. The entanglement of formation for the mixed state $\rho$ is defined as the average entanglement of the pure states of the decomposition, minimized over all possible decompositions of $\rho$,

$$
E(\rho)=\min \sum_{a=1}^{M} p_{a} E\left(\left|\psi_{a}\right\rangle\right) .
$$

For the case $N=2,(2)$ can be written as

$$
\left.E(|\psi\rangle)\right|_{N=2}=h\left(\frac{1+\sqrt{1-C^{2}}}{2}\right)
$$

where $h(x)=-x \log _{2} x-(1-x) \log _{2}(1-x), C$ is called concurrence, $C(|\psi\rangle)=2 \mid a_{11} a_{22}-$ $a_{12} a_{21} \mid$. It is easily verified that $E$ is a monotonically increasing function of $C$, and hence $C$ can be also taken as a kind of measure of the entanglement. Calculating (4) is reduced to calculate the corresponding minimum of $C(\rho)=\min \sum_{a=1}^{M} p_{a} C\left(\left|\psi_{a}\right\rangle\right)$, and an analytical expression of (4) is obtained [8].

For $N \geq 3$, there is no explicit analytical expression for the entanglement of formation in general. The concurrences discussed in $[9,10]$ can be only used to judge whether a pure state 
is separable (or maximally entangled) or not $[11,12]$, since the entanglement of formation is no longer a monotonically increasing function of these concurrences.

Nevertheless, for a special class of quantum states, certain quantities (generalized concurrence) were found to simplify the calculation of the corresponding entanglement of formation [13]. Namely, if $A A^{\dagger}$ has only two non-zero eigenvalues $\lambda_{1}$ and $\lambda_{2}$, each with degeneracy $m$, then

$$
E(|\psi\rangle)=m\left(-x \log _{2} x-\left(\frac{1}{m}-x\right) \log _{2}\left(\frac{1}{m}-x\right)\right)
$$

where

$$
x=\frac{1}{2}\left(\frac{1}{m}+\sqrt{\frac{1}{m^{2}}\left(1-d^{2}\right)}\right)
$$

and $d=2 m \sqrt{\lambda_{1} \lambda_{2}}$ is a kind of generalized concurrence taking values from 0 to 1 . One easily shows that $E(|\psi\rangle)$ is a monotonically increasing function of $d$. Moreover, $E(d)$ is a convex function,

$$
\frac{\partial^{2} E}{\partial d^{2}}=\frac{\log \frac{1+\sqrt{1-d^{2}}}{1-\sqrt{1-d^{2}}}-2 \sqrt{1-d^{2}}}{\left(1-d^{2}\right)^{3 / 2} \log 4}>0, \quad \forall d \in[0,1] .
$$

From the monotonicity and convexity the entanglement of formation for a class of high dimensional mixed states has been calculated analytically [13, 14]

In the following we generalize the results in [13] to the case that $A A^{\dagger}$ has $n \geq 3$ different non-zero eigenvalues. We present the conditions allowing to derive an explicit lower bound of the entanglement of formation for such kind of arbitrary dimensional mixed states and calculate the lower bound.

Let $\lambda_{1}, \lambda_{2}, \ldots, \lambda_{n}$, each with degeneracy $m, m n \leq N$, be the non-zero eigenvalues of $A A^{\dagger} . \lambda_{i}=\lambda_{i}(u, v), i=1,2, \ldots, n$, are differentiable functions of two real variables $u$ and $v$. We define $D=m n \sqrt{\lambda_{1} \lambda_{2} \cdots \lambda_{n}}$ to be the generalized concurrence.

[Lemma]. If $\lambda_{i}=\lambda_{i}(u, v), i=1,2, \ldots, n$, satisfy the following conditions:

$$
\sum_{i} \frac{\partial \lambda_{i}}{\partial D} \log _{2} \lambda_{i}<0
$$

then $D$ is a measure of entanglement in the sense that the entanglement of formation of the corresponding pure state is a monotonically increasing function of $D$.

[Proof] The normalization condition of $|\psi\rangle, \operatorname{Tr}\left(A A^{\dagger}\right)=\sum_{i} m \lambda_{i}=1, \lambda_{i} \in\left(0, \frac{1}{m}\right)$, gives 
rise to

$$
\sum_{i} m \frac{\partial \lambda_{i}}{\partial D}=0
$$

The entanglement of formation of $|\psi\rangle$ is given by

$$
E(|\psi\rangle)=-\sum_{i} m \lambda_{i} \log _{2} \lambda_{i}
$$

From (7) and (8) we have

$$
\frac{\partial E}{\partial D}=-\sum_{i} m \log _{2} \lambda_{i} \frac{\partial \lambda_{i}}{\partial D}
$$

which is positive if the condition (6) is satisfied. Therefore $E(|\psi\rangle)$ is a monotonically increasing function of $D$.

The Lemma defines a class of pure states, for which a generalized concurrence can be still well defined. Here we have supposed that all $\lambda_{i}$ are functions of two real variables. This implies that there is only one independent variable, accounting to the normalization condition, and the condition (6) could be plausibly satisfied. The most simple case is that $\lambda_{1}=u, \lambda_{2}=v$, each with degeneracy $m$, the generalized concurrence is given by $D=$ $2 m \sqrt{u v}$, which is just the case discussed in [13], where $E(|\psi\rangle)$ is not only a monotonically increasing but also a convex function of $D, \frac{\partial^{2} E}{\partial D^{2}} \geq 0$.

As another example we consider non-zero eigenvalues of $A A^{\dagger}$ such that $\lambda_{1}=u, \lambda_{2}=u+v$, $\lambda_{3}=u+2 v$, each with degeneracy $m, u$ and $v \in \mathbb{R}$ taking values $\left(0, \frac{1}{3 m}\right)$. The generalized concurrence is given by $D=3 m \sqrt{u(u+v)(u+2 v)}$. It is straightforward to verify that $E$ is a monotonically increasing function of $D$, since

$$
\sum_{i} \frac{\partial \lambda_{i}}{\partial D} \log _{2} \lambda_{i}=\frac{1}{3 m v \sqrt{3 m}}\left(1-9 m^{2} v^{2}\right)^{1 / 2} \log _{2} \frac{1-3 m v}{1+3 m v}<0 .
$$

Due to the relation

$$
\sum_{i} \frac{1}{\lambda_{i}}\left(\frac{\partial \lambda_{i}}{\partial D}\right)^{2}+\frac{\partial^{2} \lambda_{i}}{\partial D^{2}} \ln \lambda_{i}=\frac{1}{27 m^{3} v^{3}}\left(6 m v+\ln \frac{1-3 m v}{1+3 m v}\right)<0
$$

$E$ is also a convex function of $D$.

As $E(|\psi\rangle)$ is a monotonically increasing and convex function of $D$, instead of calculating $E(\rho)$, one may calculate the minimum decomposition (in the sense of $(4)), D(\rho)=$ $\min \sum_{a=1}^{M} p_{a} D\left(\left|\psi_{a}\right\rangle\right)$, to simplify the calculations, as long as $\rho$ has all decompositions on pure states with their eigenvalues of $A A^{\dagger}$ satisfying (6) in Lemma. Nevertheless, like $E(|\psi\rangle)$, generally the expression of $D(|\psi\rangle)=m n \sqrt{\lambda_{1} \lambda_{2} \cdots \lambda_{n}}$ could be still quite complicated. 
In fact $D(|\psi\rangle)$ is an invariant under local unitary transformations. Associated with a general pure state $|\psi\rangle$ given in (1), the following quantities are invariants under local unitary transformations $[10,11]$ :

$$
I_{0}=\operatorname{Tr}\left(A A^{\dagger}\right)=\sum_{i, j=1}^{N} a_{i j} a_{i j}^{*}, \quad I_{1}=\operatorname{Tr}\left[\left(A A^{\dagger}\right)^{2}\right]=\sum_{i, j, p, q=1}^{N} a_{i p} a_{i q}^{*} a_{j q} a_{j p}^{*} .
$$

The generalized concurrence defined in [10],

$$
C_{N}=\sqrt{\frac{N}{N-1}\left(I_{0}^{2}-I_{1}\right)}=\sqrt{\frac{N}{2(N-1)} \sum_{i, j, p, q=1}^{N}\left|a_{i p} a_{j q}-a_{i q} a_{j p}\right|^{2}},
$$

where $C_{2}=C$, does have a very simple form. However, the entanglement of formation is generally not a monotonically increasing function of $C_{N}$, and $C_{N}$ can be only used to judge wether a state is separable or maximally entangled for the case $N \geq 3$. If the generalized concurrence $D=m n \sqrt{\lambda_{1} \lambda_{2} \cdots \lambda_{n}}$ satisfies the Lemma and can be further expressed as $D=\frac{m n}{\sqrt{2}} \sqrt{I_{0}^{2}-I_{1}}$, the calculation of the corresponding entanglement of formation would be greatly simplified.

Let $\Psi$ denote the set of all pure states of the form (1) such that i) the Lemma is satisfied; ii) the entanglement of formation is a convex function of $D$, i.e., $\sum_{i} \frac{1}{\lambda_{i}}\left(\frac{\partial \lambda_{i}}{\partial D}\right)^{2}+\frac{\partial^{2} \lambda_{i}}{\partial D^{2}} \ln \lambda_{i}<0$; iii) $D=m n \sqrt{\lambda_{1} \lambda_{2} \cdots \lambda_{n}}=\frac{m n}{\sqrt{2}} \sqrt{I_{0}^{2}-I_{1}}$, that is, $\prod_{i} f_{i}(u, v)=\frac{1}{2 m}\left[(m-1)+m^{2} \sum_{i \neq j} f_{i}(u, v) f_{j}(u, v)\right]$. We call a mixed state $\rho$ given by (3) $D$-computable if all the decompositions of $\rho$ on pure states belonging to $\Psi$.

Due to the conditions i) and ii), for a $D$-computable state $\rho$, calculating $E(\rho)$ is then reduced to the calculation of the corresponding minimum of $D(\rho)=\min \sum_{a=1}^{M} p_{a} D\left(\left|\psi_{a}\right\rangle\right)$, which simplifies the calculation if $D\left(\left|\psi_{a}\right\rangle\right)$ has a simpler expression than $E\left(\left|\psi_{a}\right\rangle\right)$. The condition iii) guarantees that $D$ is a quadratic form of the entries of the matrix $A$ and can be expressed in the form of $D=\left|\left\langle\psi \mid S \psi^{*}\right\rangle\right|$ in terms of a suitable matrix $S$, which allows us to find an explicit analytical expression of the entanglement of formation in a way similar to the one used in [8] and [13]. It generalizes the results in $[13,14]$ where the case that $A A^{\dagger}$ has two non-zero eigenvalues is considered.

Let $S^{i p j q}$ be a symmetric $N^{2} \times N^{2}$ matrix whose elements are all zero except for

$$
\begin{aligned}
& S_{p+N(i-1), q+N(j-1)}=S_{q+N(j-1), p+N(i-1)}=1, \\
& S_{q+N(i-1), p+N(j-1)}=S_{p+N(j-1), q+N(i-1)}=-1,
\end{aligned}
$$


where $i, j, p, q=1, \ldots, N$. Let $\Lambda_{1}^{i p j q}, \Lambda_{2}^{i p j q}, \Lambda_{3}^{i p j q}$ and $\Lambda_{4}^{i p j q}$, in decreasing order, be the eigenvalues of the rank four Hermitian matrix $\sqrt{\sqrt{\rho} S^{i p j q} \rho^{*} S^{i p j q} \sqrt{\rho}}$.

[Theorem]. For a $D$-computable state $\rho$, the minimum decomposition of the generalized concurrence $D(\rho)$, i.e. the average generalized concurrence of the pure states of the decomposition, minimized over all decompositions of $\rho$, is given by

$$
\frac{m n}{4}\left[\sum_{i, j, p, q=1}^{N}\left(\Lambda_{1}^{i p j q}-\Lambda_{2}^{i p j q}-\Lambda_{3}^{i p j q}-\Lambda_{4}^{i p j q}\right)^{2}\right]^{\frac{1}{2}} .
$$

[Proof]. Let $r$ be the rank of $\rho$ and $\left|v_{k}\right\rangle, k=1, \ldots, r$, be a complete set of orthogonal eigenvectors corresponding to the nonzero eigenvalues of $\rho$, such that $\left\langle v_{k} \mid v_{k}\right\rangle$ is equal to the $k$ th eigenvalue. Other decomposition $\left\{\left|w_{k}\right\rangle\right\}$ of $\rho$ can then be obtained through unitary transformations:

$$
\left|w_{k}\right\rangle=\sum_{l=1}^{r} U_{k l}^{*}\left|v_{l}\right\rangle
$$

where $U$ is a $t \times t$ unitary matrix, $t \geq r$. We have $\left\langle w_{k} \mid S^{i p j q} w_{l}^{*}\right\rangle=\left(U \tau^{i p j q} U^{T}\right)_{k l}$, where the matrix $\tau^{i p j q}$ is defined by $\tau_{k l}^{i p j q}=\left\langle v_{k} \mid S^{i p j q} v_{l}^{*}\right\rangle$. As the matrix $S^{i p j q}$ is symmetric, $\tau^{i p j q}$ is also symmetric and can always be diagonalized by a unitary matrix $U$ such that $U \tau^{i p j q} U^{T}=$ $\operatorname{diag}\left(\Lambda_{1}^{i p j q}, \ldots, \Lambda_{r}^{i p j q}\right)[15]$. The diagonal elements $\Lambda_{\alpha}^{i p j q}, \alpha=1, \ldots, r$, in decreasing order, can always be made to be real and non-negative. They are also the eigenvalues of the Hermitian matrix $R \equiv \sqrt{\sqrt{\rho} S^{i p j q} \rho^{*} S^{i p j q} \sqrt{\rho}}$. The matrix $S^{i p j q}$ has $N^{2}-4$ rows and $N^{2}-4$ columns that are identically zero. It can be seen that the corresponding rows and columns of matrix $S^{i p j q} \rho^{*} S^{i p j q}$ are identically zero as well. Thus the $N^{2} \times N^{2}$ Hermitian matrix $S^{i p j q} \rho^{*} S^{i p j q}$ has a rank no greater than four. It follows that the Hermitian matrix $\sqrt{\sqrt{\rho} S^{\text {ipjq }} \rho^{*} S^{i p j q} \sqrt{\rho}}$ is ranked at most four: at least $\Lambda_{5}^{i p j q}, \Lambda_{6}^{i p j q}, \ldots, \Lambda_{r}^{i p j q}$ are zero.

There always exits a decomposition consisting of states $\left|w_{k}\right\rangle, k=1, \ldots, r$, such that $\left\langle w_{k} \mid S^{i p j q} w_{l}^{*}\right\rangle=\Lambda_{k}^{i p j q} \delta_{k l}$. Set $\left|y_{1}\right\rangle=\left|w_{1}\right\rangle,\left|y_{l}\right\rangle=i\left|w_{l}\right\rangle$ for $l=2, \ldots, r$. Any decomposition can be written in terms of the states $\left|y_{k}\right\rangle$ via the equation $\left|z_{k}\right\rangle=\sum_{l=1}^{r} V_{k l}^{*}\left|y_{l}\right\rangle$, where $V$ is a $t \times r$ matrix whose $r$ columns are orthonormal vectors.

Denote $d_{i p j q} \equiv\left|\left\langle\psi \mid S^{i p j q} \psi^{*}\right\rangle\right|=2\left|a_{i p} a_{j q}-a_{i q} a_{j p}\right|$. The average $d_{i p j q}$ of a general decomposition is given by

$$
\left\langle d_{i p j q}\right\rangle=\sum_{k}\left|\left(V Y V^{T}\right)_{k k}\right|=\sum_{k}\left|\sum_{l}\left(V_{k l}\right)^{2} Y_{l l}\right|,
$$


where $Y$ is the real diagonal matrix defined by $Y_{k l}=\left\langle y_{k} \mid S^{i p j q} y_{l}^{*}\right\rangle$. Using the fact that $\sum_{k}\left|\left(V_{k l}\right)^{2}\right|=1$, one gets

$$
\left\langle d_{i p j q}\right\rangle \geq\left|\sum_{k l}\left(V_{k l}\right)^{2} Y_{l l}\right| \geq \Lambda_{1}^{i p j q}-\Lambda_{2}^{i p j q}-\Lambda_{3}^{i p j q}-\Lambda_{4}^{i p j q}
$$

Since

$$
D(|\psi\rangle)=\frac{m n}{2} \sqrt{\sum_{i, j, p, q=1}^{N}\left|a_{i p} a_{j q}-a_{i q} a_{j p}\right|^{2}}=\frac{m n}{4} \sqrt{\sum_{i, j, p, q=1}^{N} d_{i p j q}^{2}}
$$

using the Cauchy-Schwarz inequality,

$$
\sum_{a=1}^{M}\left(\sum_{i, j, p, q=1}^{N}\left|\left\langle\psi_{a}\left|S^{i p j q}\right| \psi_{a}^{*}\right\rangle\right|^{2}\right)^{\frac{1}{2}} \geq\left[\sum_{i, j, p, q=1}^{N}\left(\sum_{a=1}^{M}\left|\left\langle\psi_{a}\left|S^{i p j q}\right| \psi_{a}^{*}\right\rangle\right|\right)^{2}\right]^{\frac{1}{2}},
$$

we get

$$
\langle D(\rho)\rangle \geq \frac{m n}{4}\left(\sum_{i, j, p, q=1}^{N}\left\langle d_{i p j q}\right\rangle^{2}\right)^{\frac{1}{2}} .
$$

Therefore the minimum decomposition of the generalized concurrence $D(\rho)$ is given by

$$
\frac{m n}{4}\left[\sum_{i, j, p, q=1}^{N}\left(\Lambda_{1}^{i p j q}-\Lambda_{2}^{i p j q}-\Lambda_{3}^{i p j q}-\Lambda_{4}^{i p j q}\right)^{2}\right]^{\frac{1}{2}} .
$$

Due to convex relation between $E(|\psi\rangle)$ and $D(|\psi\rangle)$, the entanglement of formation of $\rho$ is given by $E(D(\rho))$.

As a simple example we consider a class of pure states on $3 \times 3$ (which is not the case in $[13,14])$ with the matrix $A$ given by

$$
A=\left(\begin{array}{lll}
a_{11} & a_{12} & a_{13} \\
a_{21} & a_{22} & a_{23} \\
a_{21} & a_{22} & a_{23}
\end{array}\right)
$$

The matrix $A A^{\dagger}$ has two non-zero eigenvalues $\lambda_{1}$ and $\lambda_{2}$ satisfying

$$
\lambda_{1} \lambda_{2}=2\left(\left|a_{11} a_{22}-a_{12} a_{21}\right|^{2}+\left|a_{11} a_{23}-a_{13} a_{21}\right|^{2}+\left|a_{12} a_{23}-a_{13} a_{22}\right|^{2}\right) .
$$

It is directly verified that all pure states given by (14) belong to $\Psi$.

For all mixed states $\rho$ with decompositions on pure states (14) (it is easily seen that once $\rho$ has a decomposition with all the pure states given by (14), then all other kinds of decompositions belong to $\Psi$ too), from the theorem the lower bound of the generalized concurrence $D(\rho)$ is given by

$\sqrt{2}\left[\left(\Lambda_{1}^{1122}-\Lambda_{2}^{1122}-\Lambda_{3}^{1122}-\Lambda_{4}^{1122}\right)^{2}+\left(\Lambda_{1}^{1123}-\Lambda_{2}^{1123}-\Lambda_{3}^{1123}-\Lambda_{4}^{1123}\right)^{2}+\left(\Lambda_{1}^{1223}-\Lambda_{2}^{1223}-\Lambda_{3}^{1223}-\Lambda_{4}^{1223}\right)^{2}\right]^{\frac{1}{2}}$. 
We have studied the entanglement of formation for higher dimensional quantum mixed states. It has been shown that under certain conditions the entanglement of formation is a monotonically increasing and convex function of a generalized concurrence. For a class of ( $D$-computable) arbitrary dimensional mixed states an explicit lower bound of the entanglement of formation is derived. The generalized concurrence defined in this note is a generalization of the one in [13]. However, as we imposed on the condition $\prod_{i} f_{i}(u, v)=$ $\frac{1}{2 m}\left[(m-1)+m^{2} \sum_{i \neq j} f_{i}(u, v) f_{j}(u, v)\right]$ in calculating the lower bound of the entanglement of formation, the $D$-computable states here only cover a part of the $d$-computable states in [14]. In fact, in order to get the lower bound of the concurrence by using the method above,

it is not necessary for $D$ to be of the form $\sum_{i, j, p, q=1}^{N}\left|a_{i p} a_{j q}-a_{i q} a_{j p}\right|^{2}$, but a form of $\sum|F(a)|^{2}$ would be enough, where $F(a)$ stands for an arbitrary quadratic polynomial of the elements $a_{i j}$.

We have assumed that $\lambda_{i} \neq 0, i=1, \ldots n$, in our theorem. For $n>1$, all the pure states in $\Psi$ are entangled. Therefore the $D$-computable states are not separable in this case, as all possible decompositions, including the minimum decomposition of the entanglement of formation are in $\Psi$. One can check if these states are bound entangled by simply checking whether their are PPT (positive partial transposition) or not.

\section{References}

[1] C. H. Bennet, Phys. Scr. T76, 210 (1998).

[2] M. Nielsen and I. Chuang, Quantum Computation and Quantum Information (Cambridge University Press, Cambridge, England, 2000).

[3] C. H. Bennett, G. Brassard, S. Popescu, B. Schumacher, J. A. Smolin, and W. K.Wootters, Phys. Rev. Lett. 76, 722 (1996);

C. H. Bennett, D. P. DiVincenzo, J. A. Smolin, and W. K. Wootters, Phys. Rev. A 54, 3824 (1996).

[4] C.H. Bennett, H.J. Bernstein, S. Popescu, and B. Schumacher, Phys. Rev. A 53, 2046 (1996). 
[5] V.Vedral and M. B. Plenio, Phys. Rev. A 57, 1619 (1998), and references therein. V. Vedral, Rev. Mod. Phys. 74, 197 ( 2002).

[6] M.H. Partovi, Phys. Rev. Lett. 92, 077904 (2004).

[7] B.M. Terhal, K. Gerd and K.G.H. Vollbrecht, Phys. Rev. Lett. 85, 2625 (2000).

[8] S. Hill and W.K. Wootters, Phys. Rev. Lett. 78, 5022 (1997).

W.K. Wootters, Phys. Rev. Lett. 80, 2245 (1998).

[9] A.Uhlmann, Phys. Rev. A 62, 032307 (2000).

P. Rungta, V. Bužek, C.M. Caves, M. Hillery, G.J. Milburn, Phys. Rev. A 64, (042315) (2001).

[10] S. Albererio and S.M. Fei, J. Opt. B: Quantum Semiclass. Opt. 3, 1-5(2001).

[11] S. Albeverio, S.M. Fei and D. Goswami, Phys. Lett. A, 91-96 (2001).

[12] S.M. Fei, X.H. Gao, X.H. Wang, Z.X. Wang and K. Wu, Phys. Lett. A 300, 559$566(2002)$.

[13] S.M. Fei, J. Jost, X.Q. Li-Jost and G.F. Wang, Phys. Lett. A 310, 333-338(2003).

[14] S.M. Fei and X.Q. Li-Jost, Rep. Math. Phys. 53, 195-210(2004).

[15] R.A. Horn and C.R. Johnson, Matrix Analysis, Cambridge University Press, New York, 1985. 